\title{
FOARS: FLUTE Based Obstacle-Avoiding Rectilinear Steiner Tree Construction*
}

\author{
Gaurav Ajwani \\ Department of Electrical and \\ Computer Engineering \\ lowa State University \\ Ames, IA 50011 \\ gajwani@iastate.edu
}

\author{
Chris Chu \\ Department of Electrical and \\ Computer Engineering \\ lowa State University \\ Ames, IA 50011 \\ cnchu@iastate.edu
}

\author{
Wai-Kei Mak \\ Department of Computer \\ Science \\ National Tsing Hua University \\ Hsinchu, Taiwan 300 R.O.C. \\ wkmak@cs.nthu.edu.tw
}

\begin{abstract}
Obstacle-avoiding rectilinear Steiner minimal tree (OARSMT) construction is becoming one of the most sought after problems in modern design flow. In this paper we present FOARS, an algorithm to route a multi-terminal net in the presence of obstacles. FOARS is a top down approach which includes partitioning the initial solution into subproblems and using obstacle aware version of Fast Lookup Table based Wirelength Estimation (OA-FLUTE) at a lower level to generate an OAST followed by recombining them with some backend refinement. To construct an initial connectivity graph FOARS uses a novel obstacle-avoiding spanning graph (OASG) algorithm which is a generalization of Zhou's spanning graph algorithm without obstacle [1]. FOARS has a run time complexity of $\mathrm{O}(n \log n)$. Our experimental results indicate that it outperforms Lin et al. [2] by $2.3 \%$ in wirelength. FOARS also has $20 \%$ faster run time as compared with Long et al. [3], which is the fastest solution till date.
\end{abstract}

\section{Categories and Subject Descriptors}

B.7.2 [Hardware, Integrated Circuits, Design Aids]: Placement and Routing

\section{General Terms}

Algorithms, Design, Performance, Theory

\section{Keywords}

Physical Design, Routing, Spanning Graph, RSMT

\section{INTRODUCTION}

\footnotetext{
${ }^{*}$ This work was partially supported by National Science of Council of Taiwan under grant NSC 98-2220-E-007-031, IBM Faculty Award, and NSF under grant CCF-0540998.
}

Permission to make digital or hard copies of all or part of this work for personal or classroom use is granted without fee provided that copies are not made or distributed for profit or commercial advantage and that copies bear this notice and the full citation on the first page. To copy otherwise, to republish, to post on servers or to redistribute to lists, requires prior specific permission and/or a fee.

ISPD'10, March 14-17, 2010, San Francisco, California, USA.

Copyright 2010 ACM 978-1-60558-920-6/10/03 ...\$5.00.
With the advent of re-usability using Intellectual Property (IP) sharing, the chip in today's design is completely packed with fixed blocks such as IP blocks, macros, etc. Routing of multi-terminal nets in the presence of obstacles has become a quintessential part of the design and has been studied by many (e.g., [2-12]). As pointed out by Hwang [13], in the absence of obstacles multi-terminal net routing corresponds to the rectilinear Steiner minimal tree problem which is NPcomplete. The presence of obstacles in the region makes multi-terminal routing problem even harder.

In this work, we develop a new $O(n \log n)$ time algorithm called FOARS for OARSMT generation by leveraging FLUTE [14]. FLUTE is a very fast and robust tool for the rectilinear Steiner minimal tree problem without obstacle. It is widely used in many recent academic global routers. FLUTE by its design cannot handle obstacles. A simple strategy to generate an OARSMT would be to call FLUTE once and legalize the edges intersecting with obstacles. Unfortunately, the OARSMT obtained by such a simple strategy can be far from optimal. A better strategy is to break the Steiner tree produced by FLUTE on overlapping obstacles, recursively call FLUTE for local optimization, and then combine all locally optimized subtrees at the end. However, as the number of pins increases or if the routing region is severely cluttered with obstacles, the quality of the solution produced will degrade because it lacks a global view of the problem. To tackle this, we propose a partitioning algorithm with a global view of the problem at the top level to divide the problem into smaller instances that can be effectively handled.

To guide the partitioning algorithm, we propose to use a sparse obstacle-avoiding spanning graph (OASG) to capture the proximity information amongst the pins and corners of obstacles. Three categories of graph have been used to capture the proximity information during OARSMT construction in the past. $[4,6,7,11]$ all use the escape graph. [10] utilizes a Delaunay triangulation based graph. Both the escape graph and Delaunay triangulation based graph contain $O\left(n^{2}\right)$ edges, where $n$ is the total number of pins and obstacle corners. $[2,3,5,8,9]$ are based on various forms of obstacle-avoiding spanning graphs. Shen et al. [5] proposed a form of OASG that only contains a linear number of edges which is also adopted in [8]. Later Lin et al. [2] proposed adding missing "essential edges" to Shen's OASG. Unfortunately, it increases the number of edges to $O\left(n^{2}\right)$ in the worst case $(O(n \log n)$ in practice $)$ and hence the time complexity of later steps of OARSMT construction is increased to a 
large extent. In view of that, Long et al. [3,9] proposed a quadrant approach to generate an OASG with a linear number of edges. But as we will see later, the OASG generated by Long's approach is not ideal. In this paper, we present a novel octant approach to generate an $O(n)$-edge OASG with more desirable properties.

Different from $[2,3,5,9]$ which directly use an OASG to construct an OARSMT, we only use an OASG to guide the partitioning and construct our final OARSMT using FLUTE. We note that a shortcoming of constructing an OARSMT from an OASG directly is that it tends to follow obstacle boundaries and make detours towards obstacle corners. This makes it easier to lead to congestion when routing many nets in a design. (Adding essential edges as in [2] will help but will result in $O\left(n^{2}\right)$ edges as an escape graph.) On the other hand, since we only utilize the OASG to guide our partitioning and use FLUTE for local optimization, the OARSMT thus constructed will follow an obstacle boundary only when absolutely necessary. In addition, the OASG generated by our proposed octant approach has a linear number of edges like Long's [3,9] and possesses other desirable properties not found in Long's OASG. For example, our OASG is guaranteed to contain at least one minimum spanning tree in the absence of obstacle while Long's OASG does not have such a guarantee.

The rest of the paper is organized as follows. We first provide an overview of the main steps of our OARSMT construction approach in Section 2. Each main step is described in details in Sections 3 to 7. The experimental results are reported in Section 8. Finally, we give our conclusion in Section 9 .

\section{OVERVIEW OF THE ALGORITHM}

Our algorithm can be distinctly divided into the following five stages.

Stage 1: OASG Generation. First, we obtain the connectivity information between the pins and obstacle corner vertices using a novel octant OASG generation algorithm. Section 3 describes the OASG algorithm in detail.

Stage 2: OPMST Generation. Based on the OASG, we construct a minimum terminal spanning tree (MTST) using the approach mentioned in [15] and then obtain an obstacle penalized minimal spanning tree (OPMST) from the MTST. Section 4 talks about OPMST construction in detail.

Stage 3: OAST Generation. We partition the pin vertices based on the OPMST constructed in the previous step. After partitioning, we pass the subproblems to OA-FLUTE which calls FLUTE recursively to construct an obstacle-aware Steiner tree (OAST). Section 5 talks about the partitioning and OA-FLUTE in more detail.

Stage 4: OARSMT Generation. In this step, we rectilinearize the pin-to-pin connections avoiding obstacles to construct an OARSMT. Section 6 discusses OARSMT construction.

Stage 5: Refinement. To further reduce the wirelength, we perform V-shape refinement on the OARSMT. Details for it can be found in Section 7 .
Fig. 1 depicts the outputs after various stages of the algorithm.

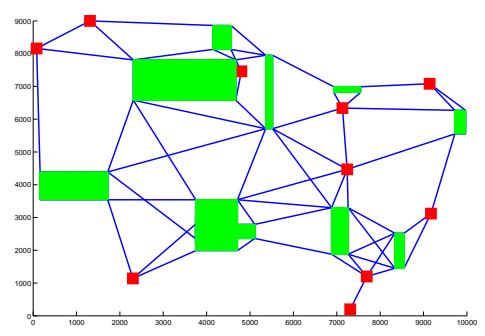

(a) OASG

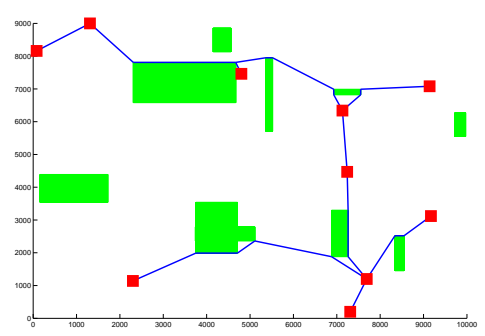

(b) MTST

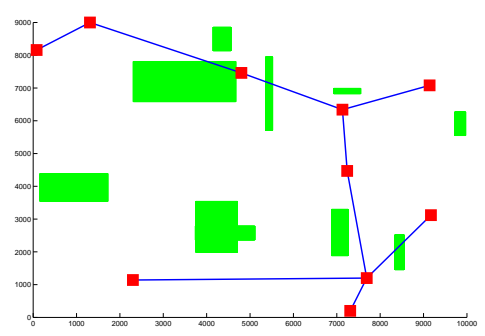

(c) OPMST

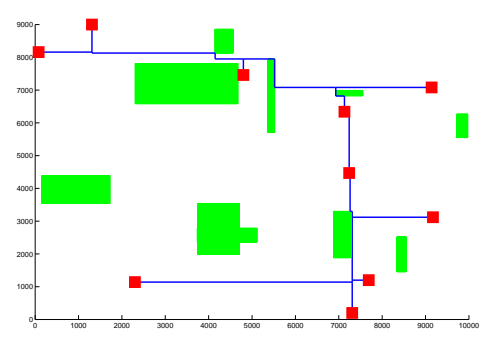

(d) OARSMT

Figure 1: Outputs at various stages for benchmark RC01

\section{OASG GENERATION}

\subsection{Previous Approaches}

Zhou et al. [1] described a spanning graph generation algorithm with $O(n)$ edges in the absence of obstacles. We prove that their approach can be seen as a special case of our obstacle-avoiding spanning graph generation algorithm. Here we start with a few definitions. 
Definition 1 Given an edge $e(u, v)$ and an obstacle $b$, e is completely blocked by $b$ if every monotonic Manhattan path connecting $u$ and $v$ intersects with a boundary of $b$.

Definition 2 Given a set of $m$ pins and $k$ obstacles, an undirected graph $G=(V, E)$ connecting all pin and corner vertices is called an $O A S G$ if none of its edges is completely blocked by an obstacle.

Although Definition 2 does not necessitate a linear number of edges for an OASG, in order to have a fast run time it is desired to limit the solution space. In the past, there have been a couple of efforts to construct an OASG with a linear number of edges. Shen et al. [5] suggested a quadrant approach in which each point can connect in four quadrants in the plane formed by horizontal and vertical line going through the point. Shen did not clearly explain their algorithm in the paper.

Long et al. [9] recently described a novel quadrant approach which is a modified version of [1] for OASG generation with a linear number of edges in $\mathrm{O}(n \log n)$ time. They suggested scanning along $\pm 45^{\circ}$ lines and maintaining an $a c$ tive vertex list, a set of vertices in the graph which are not yet connected to their nearest neighbor, similar to [1]. After scanning any vertex $v$, they search for its nearest neighbor $u$ in the active vertex list, such that the edge $(u, v)$ is not completely blocked by any obstacle in the graph. This is followed by deletion of $u$ from the list and addition of $v$ in the list.

We found that their algorithm has certain shortcomings. First, their algorithm is not symmetric, i.e., the nearest neighbor for any vertex in a quadrant is contingent upon the direction of scanning which means they have to scan along all four quadrants of a vertex in order to capture its connectivity information. Second, unlike [1] in the absence of obstacles, their algorithm cannot guarantee the presence of at least one MST in the plane. Third, their algorithm cannot handle abutting obstacles due to minor mistakes in the inequality conditions.

\subsection{Our Approach for OASG}

Looking at the above mentioned issues we conceived that rather than modifying Zhou et al's [1] approach, it will be best to simply build on their idea. Therefore, we propose an algorithm based on octant partition exhibiting uniqueness property similar to their algorithm. We reiterate the definition given in their paper. The notation $\|p q\|$ represents rectilinear distance between $p$ and $q$.

Definition 3 [1] Given a point s, a region $R$ has the uniqueness property with respect to $s$ if for every pair of points $p$, $q \in R,\|p q\|<\max (\|s p\|,\|s q\|)$. A partition of space into a finite set of disjoint regions is said to have the uniqueness property if each of its regions has the uniqueness property.

Fig. 2(a) and Fig. 2(b) describes octant partition for a pin vertex and an obstacle corner, respectively. It is proved in [1] that octant partition exhibits the uniqueness property. Imagine three points $s, p$ and $q$ such that $\|s p\|<\|s q\|$ where points $p$ and $q$ lie in $R_{i}$ of $s$. As $R_{i}$ has the uniqueness property, it implies $\|p q\|<\|s q\|$. Since the longest edge

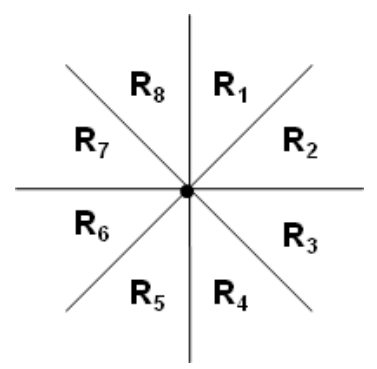

(a) Pin vertex

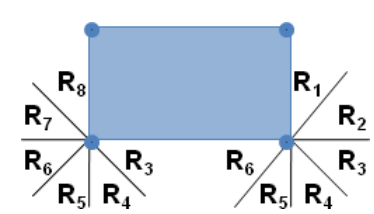

(b) Obstacle corner
Figure 2: Octant partition for a pin vertex and an obstacle corner

of any cycle should not be included in a MST, we can still guarantee that a MST exists in an OASG that does not include edge $(s, q)$.

Another interesting property of octant partition is that a contour of equidistant points from any point forms a line segment in each region. In regions $R_{1}, R_{2}, R_{5}, R_{6}$, these segments are captured by an equation of the form $x+y=c$; in regions $R_{3}, R_{4}, R_{7}, R_{8}$, they are described by the form $x-y=c$. Now this property can be exploited when we generate an obstacle-avoiding spanning graph.

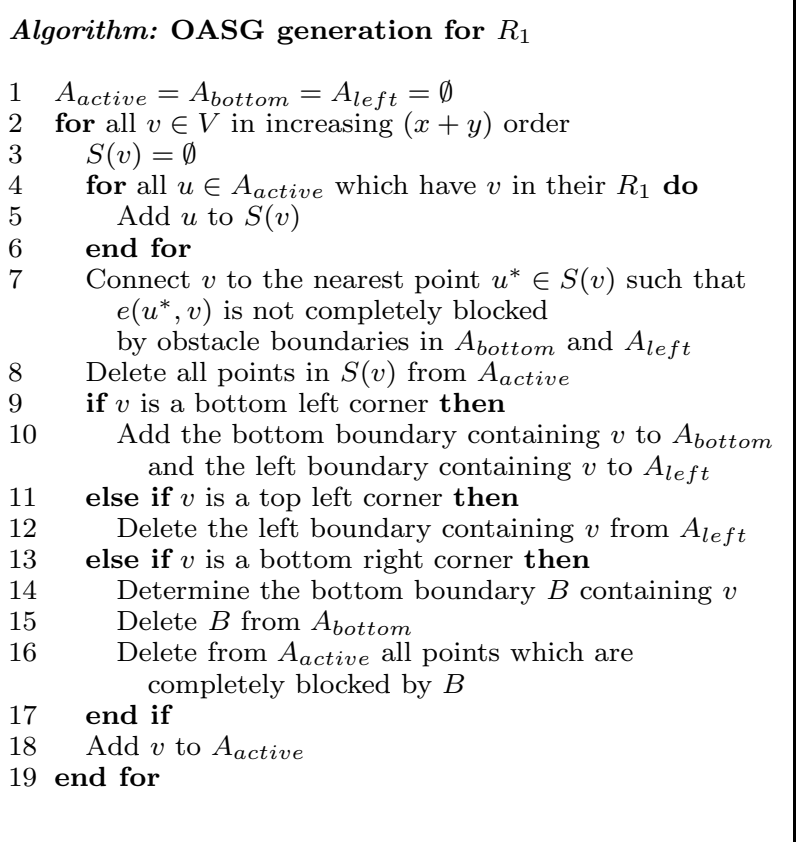

Figure 3: Pseudo code for OASG generation algorithm

The pseudo code for OASG generation for $R_{1}$ is provided in Fig. 3. As $R_{1}$ and $R_{2}$ both follow the same sweep sequence we process them together in one pass. It is worth noting that our algorithm is exactly symmetrical as it does not depend on the direction of scanning. If any point $v$ is the nearest neighbor of $u$ in $R_{1}$, it implies that $u$ is the nearest neighbor of $v$ in $R_{5}$ which reduces our sweep iterations. For any point, we only need to sweep twice to determine its connectivity information once for $R_{1} / R_{2}$ and once for 
$R_{3} / R_{4}$.

For octants $R_{1}$ and $R_{2}$, we sweep on a list of vertices in $V$ which contains both pins as well as obstacle corners with respect to increasing $(x+y)$. During sweeping we maintain an active vertex list $A_{\text {active. }}$. An active vertex is a vertex whose nearest neighbor in $R_{1}$ still needs to be discovered.

For the currently scanned vertex $v$, while looking in $R_{5}$ of $v$ we extract a subset $S(v)$ from $A_{\text {active. }}$. Any node $u$ in this subset $S(v)$ has $v$ in $R_{1}$ (lines 3 to 6 ). We connect $v$ to its nearest neighbor $u^{*}$ in $S(v)$ for which, $e\left(u^{*}, v\right)$ is not completely blocked (line 7$)$. After connecting with the nearest point we delete all the points in $S(v)$ from $A_{\text {active }}$ (line 8) and add $v$ to $A_{\text {active }}$ (line 18).

In order to determine if an edge is blocked by an obstacle, we maintain two active obstacle boundary lists, $A_{\text {bottom }}$ for the bottom boundaries and $A_{\text {left }}$ for the left boundaries. It is evident that if an edge is blocked by an obstacle in $R_{1}$, it will intersect with either its bottom or its left boundary. Next, if our scanned vertex is the bottom left corner of an obstacle, its bottom boundary is added to $A_{\text {bottom }}$ and its left boundary is added to $A_{\text {left }}$. It implies that both the left and the bottom boundaries of that obstacle become active. When we come across the top left (bottom right) corner, the corresponding boundary is removed from $A_{\text {left }}\left(A_{\text {bottom }}\right)$ implying that the left (bottom) boundary for that obstacle becomes inactive at that point (lines 12 and 15).

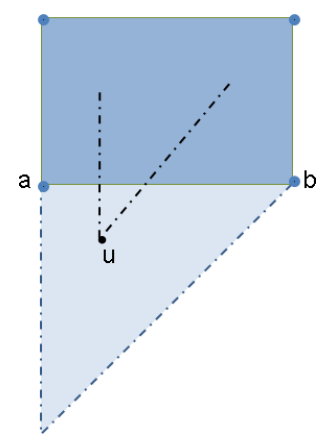

Figure 4: Completely blocked vertices

To explain lines 13 to 17 , let us refer to Fig. 4 where vertex $b$ is the bottom right corner of an obstacle. It is easy to see that if any vertex $u$ lying within the $45-45-90$ triangle shown is still in $A_{\text {active }}$ after scanning $b$, it can be removed from $A_{\text {active. }}$. Since in this case all vertices in $R_{1}$ of $u$ are completely blocked from $u$ by the obstacle.

Lemma 1 Zhou et al's algorithm [1] is a special case of our $O A S G$ generation algorithm

If we consider a case which has no obstacle, then we can simply ignore the blockage check in line 7 and lines 9 to 17 from the algorithm in Fig. 3. The resulting algorithm would be exactly the same as the algorithm in [1].

\subsection{An $O(n \log n)$ Implementation}

We first show how to perform the following fundamental operations in the OASG generation algorithm in $O(\log n)$ time: 1) Given a vertex $v$, find the subset of points in $A_{\text {active }}$ which have $v$ in their $R_{1} ; 2$ ) given an edge, check if it is completely blocked by any obstacle boundary in $A_{\text {bottom }}$ or $A_{\text {left }}$; and 3 ) given a bottom boundary of an obstacle, find all points in $A_{\text {active }}$ which are completely blocked by the boundary. We address these issues one by one in the following paragraphs.

To find the subset of $A_{\text {active }}$ which have a given point in their $R_{1}$, we need the following lemma.

Lemma 2 [1] For any two points $p$ and $q$ in the active set, we have $x_{p} \neq x_{q}$, and if $x_{p}<x_{q}$ then $x_{p}-y_{p} \leq x_{q}-y_{q}$.

We arrange $A_{v 1}$ in increasing order of x. Utilizing Lemma 2 , to find the subset of points which have $v$ in their $R_{1}$, we first find largest $x$ such that $x \leq x_{v}$. We then proceed in decreasing order of $x$ until $x-y<x_{v}-y_{v}$. Any point in between has $x \leq x_{v}$ and $x-y \geq x_{v}-y_{v}$, and hence has $v$ in its $R_{1}$. We use balanced binary search tree to implement $A_{\text {active }}$ in order to have $O(\log n)$ query operation.

An edge $e(u, v)$ formed by points $\left(x_{u}, y_{u}\right)$ and $\left(x_{v}, y_{v}\right)$ is completely blocked by a bottom obstacle boundary $(a, b)$ formed by the points $\left(x_{a}, y_{h}\right)$ and $\left(x_{b}, y_{h}\right)$, if and only if, $y_{u}<y_{h}<y_{v}, x_{a}<x_{u}$, and $x_{b}>x_{v}$. Note that at line 7 , all bottom boundaries satisfying the condition must present in the list $A_{\text {bottom. }}$. We use the balance binary search tree data structure for $A_{\text {bottom }}$ with the $y$-coordinate of a boundary as a key value. Every attempt to search for an obstacle boundary between $y_{u}$ and $y_{v}$ in $A_{b}$ takes $O(\log n)$ time. Checking if an edge is completely blocked by a left boundary can be done similarly.

To determine all the completely blocked vertices $u$ in $A_{\text {active }}$ by a horizontal boundary $(a, b)$ in line 16 , we need to check if $y_{u}<y_{h}, x_{a}<x_{u}$ and $x_{u}-y_{u}+y_{h} \leq x_{b}$ (the lightly shaded regions in Fig. 4). Since we already have $A_{\text {active }}$ as a sorted list in increasing $x$ we can check all points which lie between $x_{a}$ and $x_{b}$ and test for the above conditions to see if they are completely blocked.

The loop from line 2 to line 19 will repeat $n$ times. Lines 2-6 and $8-18$ can all be performed in $O(\log n)$ time. To analyze the total run time of line 7 , note that each $u \in V$ will only be added to some $S(v)$ at most once in line 5 . Then it will be removed from future consideration in line 8. Corresponding to each $u$ added, the blocking of one edge needs to be checked in line 7 . Hence totally $n$ edges are checked. In conclusion, the total run time of the algorithm is $O(n \log n)$.

\section{OPMST GENERATION}

\subsection{MTST Generation}

After capturing the initial connectivity amongst pin vertices, the next logical step is to extract a minimum terminal spanning tree (MTST) from the OASG that connects all pin vertices and avoid obstacles. Shen et al. [5] and Lin et al. [2] both use an indirect approach for this step. They first construct a complete graph over all pin vertices where the edge weight is the shortest path length between the two pin vertices. On this complete graph they use either Prim's or Kruskal's algorithm to obtain a MST. Although it is effective, the approach described above seems to be an overkill as it is unnecessary to construct a complete graph when we already have OASG. Back in 80's, Wu et al. [15] suggested a method using Dijkstra's and Kruskal's algorithms on a graph similar to an OASG to obtain a MTST. Recently, Long et al. [3] adopted their approach to solve the problem on the OASG. 
In this paper, we adopt the approach based on the extended Dijkstra's algorithm and the extended Kruskal's algorithm as defined in [3]. For every corner vertex in the OASG, we want to connect it with the nearest pin vertex. This can be easily done using Dijkstra's shortest path algorithm considering every pin vertex as a source. After running the extended Dijkstra's algorithm we are left with a forest of $m$ trees, $m$ being the number of pin vertices. The root of every tree in the forest obtained above is a pin vertex. In order to connect all disjoint trees we use the extended Kruskal's algorithm on the forest. A priority queue Q is used to store the weights of all possible edges termed as bridge edges in [3] which can be used for linking the trees.

Definition 4 [3] An edge $e(u, v)$ is called a bridge edge if its two end vertices belong to different terminal trees.

From Definition 4, it can be deduced that if each tree was a single vertex in the graph then bridge edges will be the edges connecting these vertices and we can use Kruskal's algorithm to obtain a MST in such a graph. The extended Kruskal's algorithm is simply an extended version of the original Kruskal's algorithm tailored to obtain a MST in a forest. It is important to note that in case we do not have any obstacle, the extended Dijkstra's algorithm will not make any change in the graph and the extended Kruskal will simply work on a spanning graph.

\subsection{OPMST Construction}

We note that a sparse OASG does not always have direct connections between the pin vertices even if one is allowed. This is due to a neighboring corner vertex being nearer than the other pin vertex in the same region. These indirect detour paths are unnecessary and if not taken care of can lead to a significant loss of quality. We note that the algorithm proposed by [3] failed to address this issue. On the other hand, we address this problem by constructing an obstacle penalized minimal spanning tree (OPMST) from the MTST by removing all the corner vertices and storing detour information as the weight of an edge.

To construct an OPMST, we follow a simple strategy. For any corner vertex $v$, we find the nearest neighboring pin vertex $u$. We connect all the pin vertices originally connected with $v$ to $u$ and delete $v$. We update their weights as their original weight plus the weight of $e(u, v)$. This method guarantees that in case we have a major detour between two pin vertices due to an obstacle, the weight of that edge will corroborate this fact. In other words we can say that the edge would be penalized for the obstacles in its path.

\section{OAST GENERATION}

This step differentiates our algorithm from [2,3,5,9]. We exploit the extremely fast and efficient Steiner tree generation capability of FLUTE [14] for low degree nets. In order to embed FLUTE in our problem we designed an obstacle aware version of FLUTE, OA-FLUTE. As OA-FLUTE is less efficient for high degree nets and dense obstacle region, we partition a high degree net into subnets guided by the OPMST obtained from the previous step. The subproblems obtained after partitioning are passed on to OA-FLUTE for obstacle aware topology generation. It is termed as obstacle aware because the nodes of the tree are placed in their appropriate location considering obstacles around them.

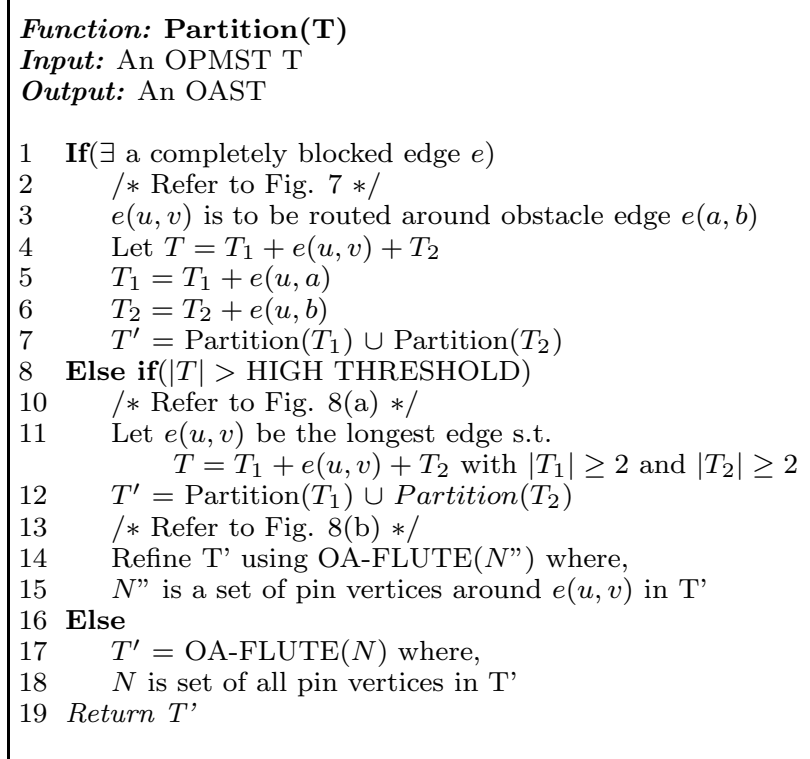

Figure 5: Pseudo code for the Partition function

Fig. 5 and Fig. 6 describe the pseudo codes for the Partition and OA-FLUTE functions. It is evident that both functions are recursive functions. Let us first explain the Partition function.

\subsection{Partition}

The input to the Partition function is an OPMST obtained from the last step and the output is an obstacle-aware Steiner tree (OAST). An OAST is a Steiner tree in which the Steiner nodes have been placed considering the obstacles present in the routing region to minimize the overall wirelength. The following two criteria are set for partitioning pin vertices. The first criterion is to determine if any edge is completely blocked by an obstacle. The second criterion is to check if the size of OPMST is more than the HIGH THRESHOLD defined.

As can be clearly seen in Fig. 7 that for an overlap free solution, we have to route around the obstacle. Therefore, it seems logical to break the tree at edge $(u, v)$. We know that $O A-F L U T E$ can efficiently construct a tree when the number of nodes is less than the HIGH THRESHOLD value. If the size of the tree is still more than the HIGH THRESHOLD after breaking at the blocking obstacles, we need to break the tree further. In this case, we look for the edge with the largest weight on the tree and delete that edge, refer to Fig. $8(\mathrm{a})$.

Based on the above mentioned criterion, if we break an obstacle edge, we simply include corner vertices in the tree and divide the two trees as shown in Fig. 7. Else, if we break at the edge with largest weight, we delete that edge and make sure that it does not contain any leaf of the tree as shown in Fig. 8(a).

After breaking an edge, we make recursive calls to the Partition function using two subtrees. When the size of the tree becomes less than the HIGH THRESHOLD, we pass the nodes of the tree to OA-FLUTE function. The $O A$ FLUTE function returns an OAST. After returning from 


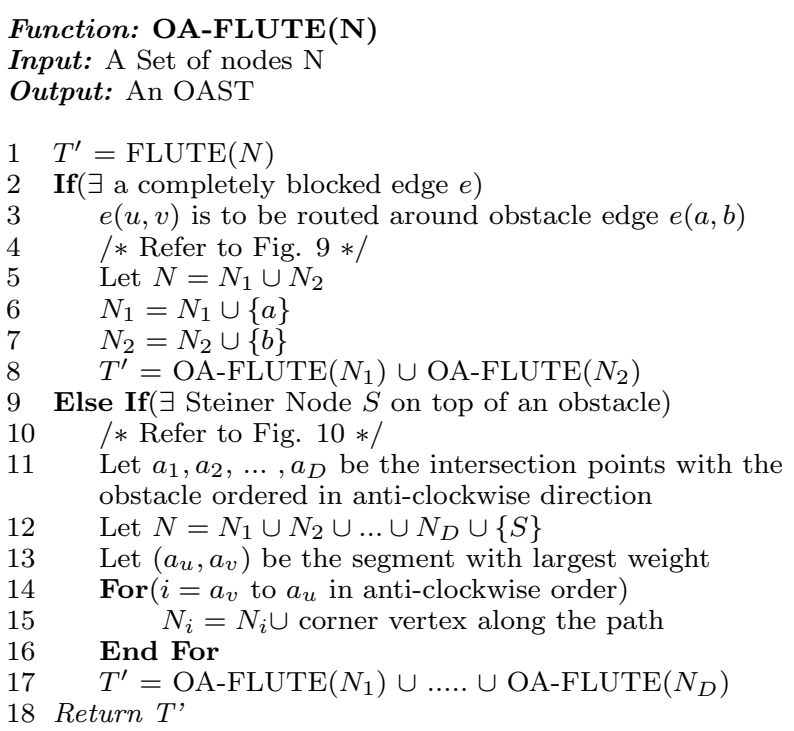

Figure 6: Pseudo code for the OA-FLUTE function

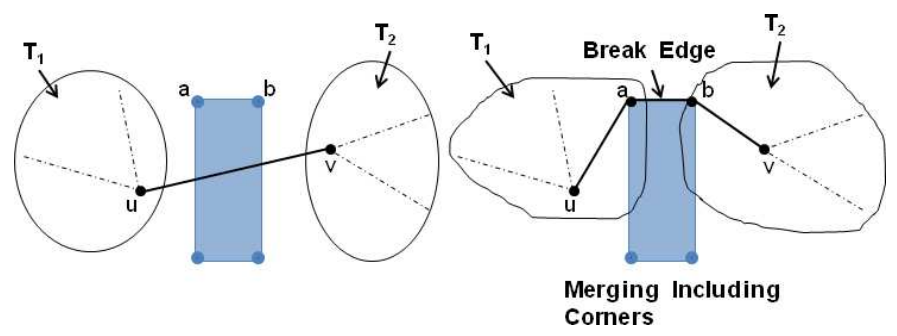

Figure 7: An example illustrating first criterion for partitioning

OA-FLUTE in Partition, if the partition was performed on an obstacle edge, we simply merge two Steiner trees using the same obstacle edge. In case the partition was performed on the longest edge, we explore an opportunity to further optimize wirelength. We merge the two trees on the longest edge and then search the region around the longest edge to extract neighboring pin vertices, refer to lines $12-15$ in Fig. 5 and Fig. 8(b). This refinement is same as the local refinement proposed in [14]. We pass this set of nodes to $O A-F L U T E$ for further optimization.

\subsection{OA-FLUTE}

The purpose of $O A-F L U T E$ function is to form an OAST. It begins by calling FLUTE on the set of input nodes. FLUTE constructs a Steiner tree without considering obstacles. This tree can have two kinds of overlap 1) an edge completely blocked by an obstacle, 2) a Steiner node on top of an obstacle. We handle both of these cases differently.

To handle the first case, refer to Fig. 9, we break the Steiner tree into two subtrees including corner points of the obstacle and make recursive calls to OA-FLUTE. We selectively prune the number of recursive calls based on the size of the tree in order to strike a balance between run-time and quality.

To handle the second case, we devised a special technique.

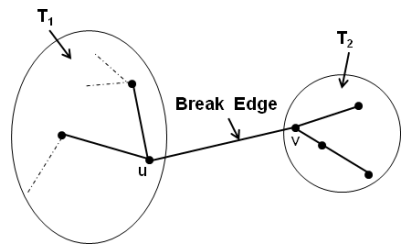

(a) Partitioning

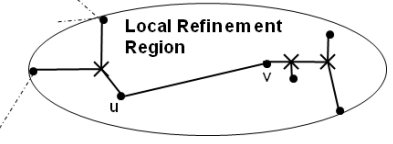

(b) Local refinement
Figure 8: An example illustrating second criterion for partitioning

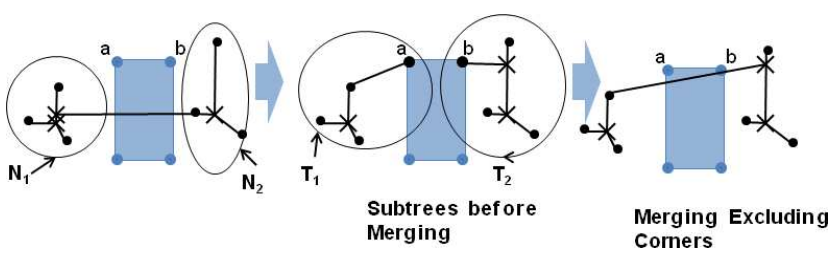

Figure 9: OA-FLUTE: An edge completely blocked by an obstacle

We pick an obstacle which has a Steiner node on top of it. For every boundary of this obstacle intersecting with the Steiner tree, we extract a set of nodes $N_{i}$ which includes the pin vertices in the tree near to that boundary. In Fig. 10 we have a single Steiner node inside the obstacle intersecting at $a_{1}, a_{2}$ and $a_{3}$, with the right, top and left boundary of the obstacle, respectively. We extract three set of pin vertices $N_{1}, N_{2}$ and $N_{3}$ from the original Steiner tree for the right, top and left boundary, respectively. The points $a_{1}, a_{2}$ and $a_{3}$ divide the obstacle outline into three segments as shown in Fig. 10. We then find the longest segment (the light shaded segment $\left(a_{3}, a_{1}\right)$ in Fig. 10). We then traverse from one endpoint of the longest segment to the other endpoint via other segments in an anti-clockwise direction, for example, from $a_{1}$ to $c_{1}$ to $a_{2}$ to $c_{2}$ to $a_{3}$ in Fig. 10. While moving along the other segments, we keep adding corner vertices to the corresponding $N_{i}$ 's e.g. $c_{1}$ gets added to both $N_{1}$ and $N_{2}$ and $c_{2}$ gets added to both $N_{2}$ and $N_{3}$. We then recursively call $O A-F L U T E$ for all $N_{i}$ 's thus formed.

As our goal with $O A-F L U T E$ is to determine befitting locations for Steiner nodes we exclude all corner vertices while merging1. Fig. 9 and Fig. 10, indicate Steiner tree after excluding corners while merging. The reason for not adding corner vertices in this step is twofold. First, it is not desirable to further restrict the solution when we already did once in Partition function. Second, we want our OA-FLUTE to be a generic function which can preserve the number of pinvertices provided to it, adding corner vertices would increase them.

\section{OARSMT GENERATION}

The OAST obtained from last step does not guarantee that rectilinear path for a pin-to-pin connection is obstacle free. In this step, we rectilinearize every pin-to-pin connection avoiding obstacles to generate an OARSMT. For every Manhattan connection between two pins we can have two Lshape paths. On the basis of the obstacles inside the bounding box formed by an edge, we can divide all the possible scenarios into four categories: 1) both L-paths are clean 2) 


\begin{tabular}{|c|c|c|c|c|c|c|c|c|c|c|c|}
\hline \multirow[b]{2}{*}{ Benchmark } & \multirow[b]{2}{*}{$\mathrm{m}$} & \multirow[b]{2}{*}{$\mathrm{k}$} & \multicolumn{5}{|c|}{ Wirelength } & \multicolumn{4}{|c|}{ Run time(s) } \\
\hline & & & $\operatorname{Lin}[2]$ & Long [3] & Liang [11] & Liu [12] & Ours & $\operatorname{Lin}[2]$ & Long [3] & Liang [11] & Ours \\
\hline RC01 & $\overline{10}$ & $\overline{10}$ & 2727790 & 26120 & 25980 & 26740 & 25980 & 0.00 & 0.00 & 0.01 & $\overline{00.00}$ \\
\hline $\mathrm{RC} 02$ & 30 & 10 & 42240 & 41630 & 42010 & 42070 & 42110 & 0.00 & 0.00 & 0.02 & 0.00 \\
\hline $\mathrm{RC} 03$ & 50 & 10 & 56140 & 55010 & 54390 & 54550 & 56030 & 0.00 & 0.00 & 0.00 & 0.00 \\
\hline $\mathrm{RC} 04$ & 70 & 10 & 60800 & 59250 & 59740 & 59390 & 59720 & 0.00 & 0.00 & 0.01 & 0.00 \\
\hline $\mathrm{RC} 05$ & 100 & 10 & 76760 & 76240 & 74650 & 75430 & 75000 & 0.00 & 0.00 & 0.01 & 0.00 \\
\hline RC06 & 100 & 500 & 84193 & 85976 & 81607 & 81903 & 81229 & 0.10 & 0.08 & 0.50 & 0.03 \\
\hline $\mathrm{RC} 07$ & 200 & 500 & 114173 & 116450 & 111542 & 111752 & 110764 & 0.18 & 0.09 & 0.60 & 0.04 \\
\hline $\mathrm{RC} 08$ & 200 & 800 & 120492 & 122390 & 115931 & 118349 & 116047 & 0.31 & 0.15 & 1.16 & 0.07 \\
\hline RC09 & 200 & 1000 & 117647 & 118700 & 113460 & 114928 & 115593 & 0.40 & 0.22 & 1.53 & 0.09 \\
\hline RC10 & 500 & 100 & 171519 & 168500 & 167620 & 167540 & 168280 & 0.20 & 0.03 & 0.18 & 0.02 \\
\hline RC11 & 1000 & 100 & 237794 & 234650 & 235283 & 234097 & 234416 & 0.74 & 0.06 & 0.83 & 0.04 \\
\hline $\mathrm{RC} 12$ & 1000 & 10000 & 803483 & 832780 & 761606 & 780528 & 756998 & 55.09 & 3.80 & 186.3 & 2.65 \\
\hline$\overline{\overline{\text { RT01 }}}$ & 10 & $\overline{500}$ & $\overline{22289}$ & 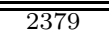 & $\overline{22231}$ & 22259 & $\overline{2191}$ & $\overline{0.03}$ & $\overline{\overline{0.06}}$ & $\overline{00.19}$ & $\overline{\overline{0.01}}$ \\
\hline RT02 & 50 & 500 & 48858 & 51274 & 47297 & 486884 & 48156 & 0.05 & 0.06 & 0.55 & 0.02 \\
\hline RT03 & 100 & 500 & 8508 & 8554 & 8187 & 8347 & 8282 & 0.10 & 0.06 & 0.21 & 0.03 \\
\hline RT04 & 100 & 1000 & 10459 & 10534 & 9914 & 10221 & 10330 & 0.22 & 0.23 & 0.37 & 0.09 \\
\hline RT05 & 200 & 2000 & 54683 & 55387 & 52473 & 53745 & 54598 & 0.96 & 0.66 & 3.18 & 0.26 \\
\hline$\overline{\text { IND1 }}$ & 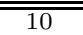 & 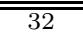 & (632 & 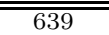 & 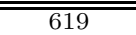 & $\overline{c 626}$ & 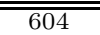 & $\overline{0.00}$ & $\overline{0.00}$ & $\overline{0.00}$ & $\overline{0.00}$ \\
\hline IND2 & 10 & 43 & 9700 & 10000 & 9500 & 9500 & 9500 & 0.00 & 0.00 & 0.00 & 0.00 \\
\hline IND3 & 10 & 59 & 632 & 623 & 600 & 600 & 600 & 0.00 & 0.00 & 0.00 & 0.00 \\
\hline IND4 & 25 & 79 & 1121 & 1130 & 1096 & 1095 & 1129 & 0.00 & 0.00 & 0.00 & 0.00 \\
\hline IND5 & 33 & 71 & 1392 & 1379 & 1360 & 1364 & 1364 & 0.00 & 0.00 & 0.00 & 0.00 \\
\hline$\overline{\overline{\text { RL01 }}}$ & 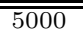 & $\overline{\overline{5000}}$ & $\overline{4992865}$ & $\overline{491855}$ & 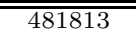 & - & $\overline{\overline{4} 483027}$ & $\overline{106.66}$ & $\overline{\overline{3.58}}$ & $\overline{27.14}$ & $\overline{\overline{3.01}}$ \\
\hline RL02 & 10000 & 500 & 648508 & 638487 & 638439 & - & 637753 & 159.09 & 1.27 & 29.45 & 1.07 \\
\hline RL03 & 10000 & 100 & 652241 & 641769 & 642380 & - & 640902 & 153.95 & 1.08 & 23.35 & 1.04 \\
\hline RL04 & 10000 & 10 & 709904 & 697595 & 699502 & - & 697125 & 195.25 & 0.97 & 22.00 & 1.39 \\
\hline RL05 & 10000 & 0 & 741697 & 728585 & 730857 & - & 728438 & 217.88 & 0.96 & 33.64 & 1.5 \\
\hline & & & (1.023) & $\overline{(1.027)}$ & $(0.995)$ & $\overline{(1.004)}$ & (1) & $891.25(78.45)$ & 13.36(1.196) & $3331.235(30)$ & $\overline{11.36(1)}$ \\
\hline
\end{tabular}

Table 1: Wirelength and run time comparison. $m$ is the number of pin vertices and $k$ is the number of obstacles. The values in the last row are normalized over our results for both wirelength as well as run time

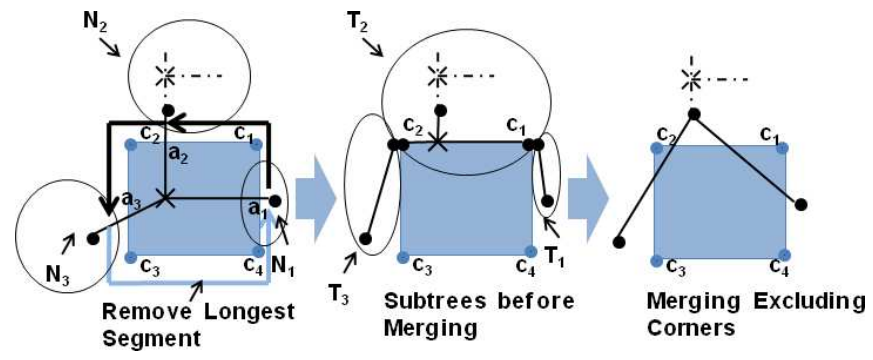

Figure 10: OA-FLUTE: Steiner node is on top of an obstacle

both L-paths are blocked by the same obstacle 3) only one L-path is blocked 4) both L-paths are blocked but not by the same obstacle. We discuss these scenarios one by one in the following paragraphs.

For the first case, even though we can rectilinearize using any L-path, we instead create a slant edge at this stage to leave the scope for improvement in $\mathrm{V}$-shape refinement. For the second case, we have no option but to go outside the bounding box and pick the least possible detour.

For the third case, we route inside the bounding box, since there exists a path. We break the edge into two sub problems on the corner of an obstacle along the blocked L-path. We recursively solve these sub problems to determine an obstacleavoiding path. If the wirelength of this path is same as the Manhattan distance between the pins, we accept the solution, else we route along the unblocked L-path. It is noteworthy that for this case we could have directly accepted the unblocked L-path. In order to create more slant edges, and hence, further scope for $\mathrm{V}$-shape refinement, we searched for a route along the blocked L-path avoiding obstacles. For the last case where both L-paths are blocked but not by the same obstacle, we determine obstacle-avoiding routes using the same recursive approach as mentioned above for both L-paths and pick the smallest one.

\section{REFINEMENT}

We perform a final V-shape refinement to improve total wirelength. This refinement includes movement of Steiner node in order to discard extra segments produced due to previous steps. The concept of refinement is similar to the one that determines a Steiner node for any three terminals. The coordinates of the Steiner node are the median value of the $\mathrm{x}$-coordinates and median value of the $\mathrm{y}$-coordinates. Fig. 11 illustrates a potential case for V-shape refinement and output after refinement. This refinement comes handy in improving the overall wirelength by $1 \%$ to $2 \%$.

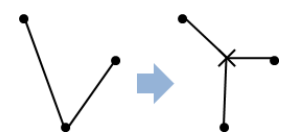

Figure 11: V-shape refinement case and refined output

\section{EXPERIMENTAL RESULTS}

We implemented our algorithm in C. The experiments were performed on a $3 \mathrm{GHz}$ AMD Athlon 64 X2 Dual Core machine. We requested for binaries from Long et al. [3], Lin et al. [2], Liang et al. [11] and ran them on our platform. We could not get binary from Liu et al. [12], which is the most recent work, on time to include in the paper. We report their results as provided in their paper. Table 1 shows Wirelength and CPU run time comparison with them. There 
are four sets of benchmarks. Five industrial test cases are from Synopsys(IND1 - IND05), twelve circuits are from [2] (RC01-RC12), five randomly generated benchmark circuits (RT01-RT05) [2] and five large benchmark circuits (RL01RL05) generated by [3]. We determined experimentally that HIGH THRESHOLD value of 20 works the best.

As shown in last row, column 4,5 and 7, of Table 1, on an average over all benchmarks, our wirelength results outperform Lin et al. [2] by $2.3 \%$ and Long et al. [3] by $2.7 \%$ and Liu et al. [12] by $0.4 \%$. But column 6 indicates that our results are $0.5 \%$ longer as compared to Liang et al. [11]. This could be attributed to the fact that they use maze routing approach.

Our results also indicate that our algorithm performs better in terms of quality in all higher order (RC07 - RC12)(RL01 - RL05) benchmarks than Liu et al. and we are just $0.1 \%$ longer than Liang et al. and 30 times more efficient in CPU run time. We believe that larger benchmarks with more number of pin vertices and more number of obstacles (similar to RC12) are more scalable in industry and we outperform all other existing approaches in these benchmarks due to our highly efficient steiner tree generation tool, OA-FLUTE.

For the run time, we are $20 \%$ faster than Long et al. [3] on average. We are 33 times faster than [11] and 88 times faster than [2]. We could not make a direct comparison between the run times of Liu et al. as we could not run their binary on our platform.

We can conclude from the above discussion that existing heuristics improve either run time or wirelength but not both. Our algorithm improves both in terms of quality and run time as compared to the algorithms [5], [2] and [3], of its kind. Also we have the best results both for wirelength and run time for higher-order benchmarks(RC12, RL01-RL05), when compared to [11] and [12] which indicates the applicability of our algorithm to industrial standard circuits.

\section{CONCLUSION}

In this paper, we have presented FOARS, an efficient algorithm to construct OARSMT based on extremely fast and efficient Steiner tree generation tool called FLUTE. We propose a novel OASG algorithm with linear number of edges. We also propose an obstacle aware version of FLUTE, which generates OAST. Our top-down partition approach empowers OA-FLUTE to handle high degree multi-terminal net and dense obstacle region. Our results indicates that our approach is the best tradeoff for quality and run time. Our experiments prove that FOARS obtains good quality solution with excellent run-time as compared with its peers.

\section{ACKNOWLEDGEMENT}

We acknowledge Lin et. al [2], Liang et. al [11] and Long et. al [3] for sending us their binaries for comparison and clearing our doubts, if any, with respect to the results.

\section{REFERENCES}

[1] Hai Zhou, Narendra V. Shenoy, and William Nicholls. Efficient minimum spanning tree construction without delaunay triangulation. In Proc. of $A S P-D A C$, pages 192-197, 2001.

[2] Chung-Wei Lin, Szu-Yu Chen, Chi-Feng Li, Yao-Wen Chang, and Chia-Lin Yang. Obstacle-avoiding rectilinear Steiner tree construction based on spanning graphs. In Proc. of IEEE Transactions on CAD of Integrated Circuits and Systems, 27(4):643-653, 2008.

[3] Jieyi Long, Hai Zhou, and Seda Ogrenci Memik. EBOARST: An efficient edge-based obstacle avoiding-rectilinear Steiner tree construction algorithm. In Proc. of IEEE Transactions on CAD of Integrated Circuits and Systems, 27(12), 2008.

[4] Yu Hu, Zhe Feng, Tong Jing, Xianlong Hong, Yang yang Ge, Xiaodong $\mathrm{Hu}$, and Guiying Yan. FORst: A 3-step heuristic for obstacle-avoiding rectilinear Steiner minimal tree construction. In Proc. of JICS, pages 107-116, 2004.

[5] Zion Shen, Chris C. N. Chu, and Ying-Meng Li. Efficient rectilinear Steiner tree construction with rectilinear blockages. In Proc. of ICCD, pages 38-44, 2005.

[6] Yu Hu, Tong Jing, Xianlong Hong, Zhe Feng, Xiaodong $\mathrm{Hu}$, and Guiying Yan. An-OARSMan: Obstacle-avoiding routing tree construction with good length performance. In Proc. of ASP-DAC, pages 630-635, 2006.

[7] Yiyu Shi, Paul Mesa, Hao Yao, and Lei He. Circuit simulation based obstacle-aware Steiner routing. In Proc. of DAC, pages 385-388, 2006.

[8] Pei-Ci Wu, Jhih-Rong Gao, and Ting-Chi Wang. A fast and stable algorithm for obstacle-avoiding rectilinear Steiner minimal tree construction. In Proc. of ASP-DAC, pages 262-267, 2007.

[9] Jieyi Long, Hai Zhou, and Seda Ogrenci Memik. An $\mathrm{O}(\mathrm{n} \log \mathrm{n})$ edge-based algorithm for obstacle-avoiding rectilinear Steiner tree construction. In Proc. of ISPD, pages 126-133, 2008.

[10] Iris Hui-Ru Jiang, Shung-Wei Lin, and Yen-Ting Yu. Unification of obstacle-avoiding rectilinear Steiner tree construction. In Proc. of SoCC, pages 127-130, 2008.

[11] Liang Li and Evangeline F. Y. Young. Obstacle-avoiding rectilinear Steiner tree construction. In Proc. of ICCAD, pages 523-528, 2008.

[12] Chih-Hung Liu, Shih-Yi Yuan, Sy-Yen Kuo, and Yao-Hsin Chou. An O(n log n) path-based obstacle-avoiding algorithm for rectilinear Steiner tree construction. In Proc. of DAC, pages 314-319, 2009.

[13] F. K. Hwang. On Steiner minimal trees with rectilinear distance. In Proc. of SIAM J. Appl. Math, 30:104-114, 1976.

[14] Chris Chu and Yiu-Chung Wong. FLUTE: Fast lookup table based rectilinear Steiner minimal tree algorithm for VLSI design. In Proc. of IEEE Transactions on CAD of Integrated Circuits and Systems, 27(1):70-83, 2008.

[15] Y. F. Wu, P. Widmayer, and C. K. Wong. A faster approximation algorithm for the Steiner problems in graphs. In Proc. of Acta Informatica, 23:223-229, 1986. 